\title{
Nomograms predicting primary lymph node metastases and prognosis for synchronous colorectal liver metastasis with simultaneous resection of colorectal cancer and liver metastases
}

\author{
Jinjie Yao", Qichen Chen", Yiqiao Deng, Jianjun Zhao, Xinyu Bi, Zhiyu Li, Zhen Huang, Yefan Zhang, \\ Jianguo Zhou, Hong Zhao, Jianqiang Cai
}

Department of Hepatobiliary Surgery, National Cancer Center/National Clinical Research Center for Cancer/Cancer Hospital, Chinese Academy of Medical Sciences and Peking Union Medical College, Beijing, China

Contributions: (I) Conception and design: H Zhao, J Cai; (II) Administrative support: J Zhao, X Bi, Z Li; (III) Provision of study materials or patients: Z Huang, Y Zhang, J Zhou; (IV) Collection and assembly of data: J Yao, Q Chen; (V) Data analysis and interpretation: J Yao, Q Chen; (VI) Manuscript writing: All authors; (VII) Final approval of manuscript: All authors.

\#These authors contributed equally to this work.

Correspondence to: Hong Zhao; Jianqiang Cai. Department of Hepatobiliary Surgery, National Cancer Center/National Clinical Research Center for Cancer/Cancer Hospital, Chinese Academy of Medical Sciences and Peking Union Medical College, Beijing 100021, China. Email: pumczhaohong@126.com; caijianqiang188@sina.com.

Background It is necessary to identify valuable predictors of primary lymph node metastasis and prognosis
for patients with synchronous colorectal cancer liver metastases (CRLM) with simultaneous resection of
colorectal cancer (CRC) and liver metastases. This study constructed nomograms especially incorporating
preoperative testing markers to predict primary lymph node metastases and prognosis in CRLM patients.
Methods: By the highest Youden index (sensitivity + 1-specificity), the optimal cut-off values of testing
markers for postoperative major complications and lymph node metastasis were identified. Multivariate
regression analysis was used to reveal independent predictors for primary lymph node metastasis,
postoperative major complications and progression-free survival (PFS). Nomograms based on independent
predictors were constructed, and the discrimination and calibration were evaluated. Results: A nomogram predicting primary lymph node metastasis was based on four risky independent predictors: American Society of Anesthesiologists (ASA) score 3-4, preoperative albumin (ALB) $<41.15$ g/L, poor differentiation and multiple liver metastases. The performance of the model was acceptable in predicting lymph node metastasis, with an area under the receiver operating characteristic curve (AUROC) of 0.655 (95\% CI: 0.591-0.739). Calibration curves and the Hosmer-Lemeshow test revealed desirable model calibration (chi-square: $13.26, \mathrm{P}=0.815$ ). In the multivariate analysis, preoperative lactate dehydrogenase $(\mathrm{LDH}) \geq 202.5 \mathrm{U} / \mathrm{L}$ [odds ratio $(\mathrm{OR})=2.084$, 95\% confidence interval $(\mathrm{CI}): 1.039-4.181, \mathrm{P}=0.039$ ] and operation time $\geq 350.5 \mathrm{~min}(\mathrm{OR}=2.848,95 \% \mathrm{CI}: 1.418-5.723, \mathrm{P}=0.003$ ) were independently associated with the presence of postoperative major complications. A nomogram predicting PFS was constructed based on poor differentiation, positive lymph node metastasis, bilobar liver distribution and R0 resection with good discrimination (C-index: 0.656 \pm 0.021$)$ and calibration.

Conclusions: This study established predictive nomograms specifically incorporating preoperative ALB and $\mathrm{LDH}$ levels for the prediction of primary lymph node metastasis and prognosis in synchronous CRLM patients with simultaneous resection, which have favourable discrimination and calibration to make individualized predictions.

Keywords: Colorectal liver metastases; nomogram; lymph node metastasis; postoperative complications; progression-free survival (PFS)

Submitted Nov 19, 2020. Accepted for publication Feb 24, 2021

doi: 10.21037/apm-20-2303

View this article at: http://dx.doi.org/10.21037/apm-20-2303 


\section{Introduction}

Colorectal cancer (CRC) is the third most common malignancy in the world (1). More than $50 \%$ of patients with CRC presented with liver metastases (2). At CRC diagnosis, synchronous liver metastases are already present in approximately $15 \%$ of patients $(3,4)$. Curative resection plays an important role in the treatment of synchronous colorectal cancer liver metastases (CRLM). For initially resectable CRLM, the timing of colorectal and liver surgery remains controversial $(5,6)$. Compared with stage resection, simultaneous resection of CRC and metastases has the advantages of requiring a single operation, lower cost, similar postoperative complication rates and even more favourable overall survival (OS) $(5,6)$. Simultaneous resection has been advocated by increasing numbers of clinicians.

The prognosis of patients with CRLM with simultaneous resection of CRC and liver metastases is still not satisfactory. Overall, 75\% (7) of patients still experience recurrence after surgery; the 5-year progression-free survival (PFS) and OS rates are only $21.2 \%$ and $46.4 \%$, respectively (8). The incidence rates of postoperative complications and major complications are approximately $47 \%$ and 12\% (9). Positive primary lymph node metastasis is a risky independent predictor for survival in CRLM patients, which leads to more unfavourable survival $(10,11)$. It is urgent to identify predictive factors of primary lymph node metastasis and prognosis for patients with synchronous CRLM with simultaneous resection of CRC and liver metastases. A nomogram, a model for predicting the risk and prognosis probability and guiding the strategies of treatment and follow-up plans, plays an increasingly important role in clinical practice. Tang et al. (12) established nomogram for predicting occurrence and OS of synchronous colorectal liver metastasis and Yan et al. (13) constructed nomogram to predict lymph node metastasis and liver metastasis in patients with early colon carcinoma. However, these studies did not consider the effect of pre-operative testing markers. Preoperative testing marker levels from preoperative blood routines have been investigated to predict the prognosis of many tumors (14-17). There is still no study investigating the role of preoperative testing markers in the prediction of primary lymph node metastasis, post-operative complications and prognosis in patients with synchronous CRLM with simultaneous resection of CRC and liver metastases. The present study firstly filled blank and constructed nomograms incorporating preoperative testing markers to help the clinicians identify high-risk patients and design individualized treatment. We presented the following article in accordance with the TRIPOD reporting checklist (available at http:// dx.doi.org/10.21037/apm-20-2303).

\section{Methods}

\section{Patients}

From January 2008 to December 2017, a total of 241 patients with CRLM were enrolled who underwent hepatectomy with simultaneous primary colorectal resection at Cancer Hospital, Chinese Academy of Medical Sciences. The study was conducted in accordance with the Declaration of Helsinki (as revised in 2013). Ethical approval was obtained from the Institutional Review Board of the Cancer Hospital, Chinese Academy of Medical Sciences (ID: NCC2019C-016). Informed consents have been obtained. The inclusion criteria were (I) histologically confirmed liver metastases of colorectal adenocarcinoma and (II) patients underwent hepatic resection with simultaneous primary colorectal resection. The exclusion criteria were (I) loss to follow-up or incomplete clinical data and (II) other malignancies. We used peripheral venipuncture to collect blood samples within one week before surgery for the evaluation of serum testing markers. Specific markers included the following: preoperative serum alanine aminotransferase (ALT) value (normal range, 9$50 \mathrm{U} / \mathrm{L}$ ), aspartate aminotransferase (AST) value (normal range, 15-10 U/L), albumin (ALB) value (normal range, 40$50 \mathrm{~g} / \mathrm{L}$ ), lactate dehydrogenase (LDH) level (normal range, 120-250 U/L), fibrinogen (FIB) value (2-4 g/L), urea level (normal range, 3.1-8.0 mmol/L) and creatinine level (normal range, $57-111 \mu \mathrm{mol} / \mathrm{L})$.

\section{Treatments}

Neoadjuvant chemotherapy (NAC) was recommended for CRLM patients with high risk factors for recurrence (18). The NAC regimen included a combination of 5-fluorouracil/ capecitabine and oxaliplatin/irinotecan with or without targeted therapy (bevacizumab and cetuximab). Patients, after the completion of NAC treatment, usually underwent liver resections within 4-6 weeks. Postoperative complications were graded according to the Clavien system, and major complications were defined as grade III or IV complications. The highest grade was used for patients who experienced any 
multiple postoperative complications.

\section{Follow-up and outcomes}

Patients were followed up at regular intervals after surgery. The first follow-up date was one month after surgery, with subsequent follow-ups every 3 months for 2 years, every 6 months between 2-5 years, and every 1 year afterwards. PFS was defined as the interval from the date of resection to progression or the last follow-up.

\section{Statistical analysis}

To analyse continuous and categorical variables, the MannWhitney $U$ test and the chi-square or Fisher's exact test were adopted, respectively. The PFS were measured by the Kaplan-Meier method and compared with the log-rank test. Youden index is optimized over the entire range of values for sensitivity and specificity, which determine the ROC space. The optimal cut-off values were identified using the highest Youden index (sensitivity + 1-specificity) (19). On the basis of the highest Youden index, the optimal cut-off values of perioperative markers for postoperative major complications and positive lymph node metastasis were determined. To identify the optimal segmentation threshold for survival curve risk stratification, an $\mathrm{X}$-tile analysis was carried out. The multivariate models retained all predictors with $\mathrm{P}<0.10$ in the univariate analysis. Through the logistic regression analysis model, multivariable analysis of the relationships between patient characteristics and postoperative major complications and positive lymph node metastasis was performed. Through the Cox regression model, multivariable analysis of survival was performed. Forward: likelihood ratio was implemented in multivariate analysis. $\mathrm{P}<0.05$ was considered statistically significant. The construction of the nomogram employed statistically meaningful independent predictors. To explore the model discrimination, the C-index and area under the receiver operating characteristic curve (AUROC) were used. To evaluate the model calibration, the calibration plots and the Hosmer-Lemeshow chi-square test were performed. Statistical analyses were performed using SPSS version 22 software (Armonk NY, USA) and R software (http://www.r-project.org).

\section{Results}

\section{Clinicopathological characteristics}

A total of 241 patients were enrolled in our study, including
87 females and 154 males with a mean age of $57.48 \pm$ 9.55 years. Among them, 135 patients $(56.0 \%)$ received NAC treatment. Primary lymph node metastasis of the primary tumour was observed in 177 patients (73.4\%). Poor differentiation of tumours was discovered in 59 patients (24.5\%). Bilobar distribution of liver metastases and extrahepatic metastases were found in 96 patients (39.8\%) and 23 patients (9.5\%), respectively. The median diameter of the largest liver metastasis was 2.5 [interquartile range (IQR), $1.5-4.0] \mathrm{cm}$, and the median number of liver metastases was 2 (IQR, 1-4). The median operation time was 340.0 (IQR, 272.5-420.0) $\mathrm{min}$, and the median intraoperative blood loss was 200 (IQR, 100-400) mL. The preoperative median carcinoembryonic antigen (CEA) value was 8.88 (IQR, 3.92-32.42) $\mathrm{ng} / \mathrm{mL}$. The preoperative median ALT and AST values were 18.0 (IQR, 12.0-24.0) U/L and 21.0 (IQR, 16.026.0) $\mathrm{U} / \mathrm{L}$, respectively. The preoperative median ALB value was 41.7 (IQR, 38.75-44.3) g/L. The preoperative median FIB value was 3.20 (IQR, 2.69-3.78) g/L. The preoperative median LDH level was 179.0 (IQR, 152.5-207.0) U/L. The preoperative median urea level was 4.6 (IQR, 3.9-5.6). The preoperative median creatinine level was 67 (IQR, 5878) $\mu \mathrm{mol} / \mathrm{L}$. The demographic and clinical characteristics of the patients are listed in Table 1.

\section{Predictors for lymph node metastasis}

In this study, patients with positive lymph node metastasis accounted for $73.4 \%$. In the univariate analysis, preoperative $\mathrm{ALB} \geq 41.15 \mathrm{~g} / \mathrm{L}(\mathrm{P}=0.021)$, poor differentiation $(\mathrm{P}=0.027)$, multiple liver metastases $(\mathrm{P}=0.007)$ and bilobar liver distribution $(\mathrm{P}=0.027)$ were notably associated with lymph node metastasis. In addition, $B M I \geq 24 \mathrm{~kg} / \mathrm{m}^{2}(\mathrm{P}=0.053)$ and American Society of Anesthesiologists (ASA) score 3-4 $(\mathrm{P}=0.083)$ showed tendencies towards lymph node metastasis. All the above-mentioned predictors $(\mathrm{P}<0.1)$ were incorporated in the multivariate analysis, and ASA score 3-4 [odds ratio $(\mathrm{OR})=3.214,95 \%$ confidence interval (CI): $1.251-8.257, \mathrm{P}=0.015]$, preoperative ALB $\geq 41.15 \mathrm{~g} / \mathrm{L}$ (OR $=0.406,95 \%$ CI: 0.214-0.772, $\mathrm{P}=0.006$ ), poor differentiation $(\mathrm{OR}=3.252,95 \% \mathrm{CI}: 1.432-7.382$, $\mathrm{P}=0.005)$ and multiple liver metastases $(\mathrm{OR}=2.521,95 \%$ CI: $1.367-4.651, \mathrm{P}=0.003)$ were independently associated with the presence of lymph node metastasis (Table 2). A nomogram with four independent predictors from the multivariate analysis was developed (Figure 1). These factors were assigned specific scores as follows: ASA score 3-4, 99; preoperative ALB $<41.15 \mathrm{~g} / \mathrm{L}, 76$; poor differentiation, 
Table 1 Characteristics of CRLM patients after liver resection

\begin{tabular}{|c|c|}
\hline Characteristics & Total $(n=241)$ \\
\hline Age, mean (SD) & $57.48(9.55)$ \\
\hline Male, n (\%) & $154(63.9)$ \\
\hline $\mathrm{BMI} \geq 24$ kg/m², n (\%) & $118(49.0)$ \\
\hline Comorbidity, n (\%) & $109(45.2)$ \\
\hline ASA score 3-4, n (\%) & $118(49.0)$ \\
\hline Preoperative CEA, median [IQR] & 8.88 [3.92-32.42] \\
\hline Preoperative ALT, median [IQR] & $18.0[12.0-24.0]$ \\
\hline Preoperative AST, median [IQR] & 21.0 [16-26.0] \\
\hline Preoperative ALB, median [IQR] & 41.7 [38.75-44.3] \\
\hline Preoperative FIB, median [IQR] & $3.20[2.69-3.78]$ \\
\hline Preoperative LDH, median [IQR] & $179.0[152.5-207.0]$ \\
\hline Preoperative Urea, media [IQR] & $4.6[3.9-5.6]$ \\
\hline Preoperative creatinine, media [IQR] & $67[58-78]$ \\
\hline Primary site in colon, $\mathrm{n}(\%)$ & $143(59.3)$ \\
\hline Right hemicolon, n (\%) & $47(19.5)$ \\
\hline Diameter of metastases, media [IQR] & $2.5[1.5-4]$ \\
\hline Number of metastases, media [IQR] & $2[1-4]$ \\
\hline Bilobar liver distribution, n (\%) & $96(39.8)$ \\
\hline Extrahepatic metastases, n (\%) & $23(9.5)$ \\
\hline Ro resection, $\mathrm{n}(\%)$ & $160(66.4)$ \\
\hline Poor differentiation, n (\%) & $59(24.5)$ \\
\hline T3-T4 stage, n (\%) & $225(93.4)$ \\
\hline Positive lymph node metastasis, $n$ (\%) & $177(73.4)$ \\
\hline KRAS mutation ${ }^{\mathrm{a}}, \mathrm{n}(\%)$ & 47 (38.2) \\
\hline Concomitant RFA, n (\%) & $31(12.9)$ \\
\hline Operation time (min), media [IQR] & $340.0[272.5-420.0]$ \\
\hline Blood loss (mL), median [IQR] & $200[100-400]$ \\
\hline Neoadjuvant chemotherapy, n (\%) & $135(56.0)$ \\
\hline Postoperative chemotherapy, n (\%) & $130(53.9)$ \\
\hline Postoperative complications, n (\%) & $109(45.2)$ \\
\hline Postoperative major complications, $\mathrm{n}(\%)$ & $45(18.7)$ \\
\hline
\end{tabular}

a, KRAS status was available in 123 patients. CRLM, colorectal cancer liver metastases; ASA, American Society of Anesthesiologists; CEA, carcinoembryonic antigen; ALT, alanine aminotransferase; AST, aspartate aminotransferase; ALB, albumin; FIB, fibrinogen; LDH, lactate dehydrogenase.
100; and multiple liver metastases, 78. The performance of the model was convincing in predicting lymph node metastasis, with an AUROC of 0.655 (95\% CI: 0.5910.739). Calibration curves and the Hosmer-Lemeshow test also revealed desirable model calibration (chi-square: 13.26, $\mathrm{P}=0.815$ ) (Figure 2).

\section{Predictors for postoperative major complications}

In all cases, the proportion of patients who underwent postoperative complications was $45.2 \%$, including 45 major complications and 64 minor complications. In the univariate analysis (Table 2), preoperative $\mathrm{LDH} \geq 202.5 \mathrm{U} / \mathrm{L}(\mathrm{P}=0.007)$, concomitant radiofrequency ablation (RFA) $(\mathrm{P}=0.042)$, operation time $\geq 350.5 \mathrm{~min}(\mathrm{P}=0.001)$ and intraoperative blood loss $\geq 450 \mathrm{~mL}(\mathrm{P}=0.040)$ were markedly correlated with postoperative major complications. Whereafter, the above predictors with statistical significance $(\mathrm{P}<0.05)$ were included in the multivariate analysis, and preoperative $\mathrm{LDH}$ $\geq 202.5$ U/L (OR $=2.084,95 \%$ CI: 1.039-4.181, $\mathrm{P}=0.039$ ) and operation time $\geq 350.5 \mathrm{~min}(\mathrm{OR}=2.848,95 \% \mathrm{CI}$ : $1.418-5.723, \mathrm{P}=0.003)$ were independently related to the existence of postoperative major complications (Table 3).

\section{Prognostic factors for PFS}

Of all patients, $77.18 \%$ experienced tumour progression. The median PFS was 10.0 (IQR, 8.7-11.3) months, and the 1 - and 3 -year PFS rates were $42.7 \%$ and $21.1 \%$, respectively. In the univariable analysis, poor differentiation, positive lymph node metastasis, multiple liver metastases, bilobar liver distribution, extrahepatic metastases, concomitant RFA, non-R0 resection and operation time $\geq 350.5$ min were relevant to decreased $\mathrm{PFS}(\mathrm{P}<0.05)$. Four independent prognostic factors for PFS were identified in the multivariable analysis: poor differentiation [hazard ratio $(\mathrm{HR})=1.427,95 \% \mathrm{CI}: 1.027-1.982, \mathrm{P}=0.034$, positive lymph node metastasis (HR $=1.624,95 \%$ CI: $1.125-2.344$, $\mathrm{P}=0.010)$, bilobar liver distribution $(\mathrm{HR}=1.478$, 95\% CI: 1.086-2.012, $\mathrm{P}=0.013)$ and $\mathrm{R} 0$ resection $(\mathrm{HR}=0.706$, 95\% CI: 0.515-0.967, P=0.030) (Table 4). Furthermore, we also constructed a prognostic nomogram for PFS with point scales via the above four independent prognostic factors (Figure 3). These factors were allocated specific scores as follows: poor differentiation, 74; positive lymph 
Table 2 Prognostic factors for major complications in CRLM patients after liver resection

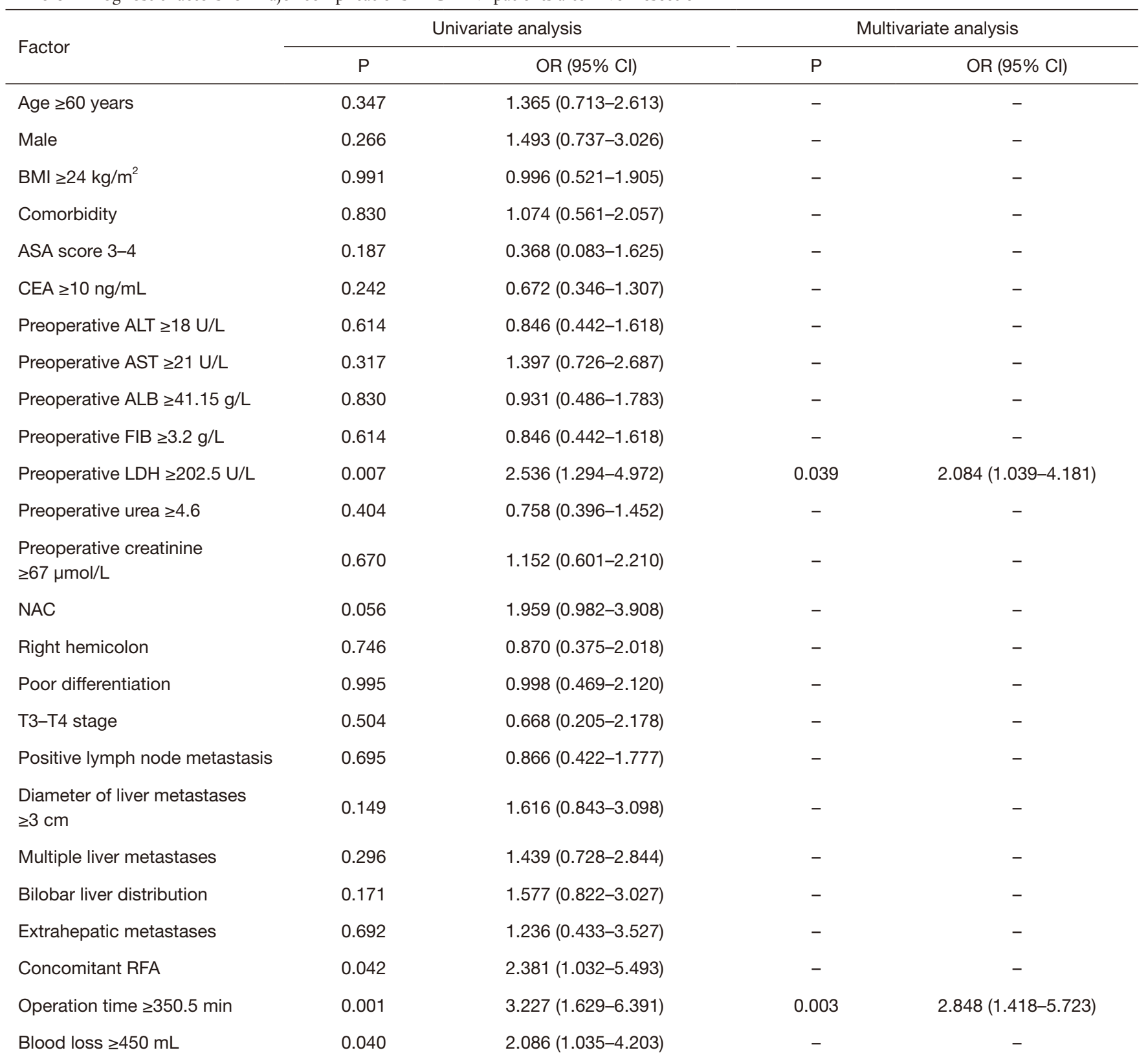

CRLM, colorectal cancer liver metastases; ASA, American Society of Anesthesiologists; CEA, carcinoembryonic antigen; ALT, alanine aminotransferase; AST, aspartate aminotransferase; ALB, albumin; FIB, fibrinogen; LDH, lactate dehydrogenase; NAC, neoadjuvant chemotherapy; RFA, radiofrequency ablation.

node metastasis, 100; bilobar liver distribution, 80; and R0 resection, 72. The prediction ability of this model for PFS was satisfactory, and the C-statistic was $0.656 \pm 0.021$. A calibration plot for the probability of PFS at 1,3 and 5 years (Figure 4) demonstrated good calibration between the predictions by the nomogram and the actual observations.

\section{Discussion}

This study established nomograms specifically considering 
Points
ASA score
Preoperative ALB level
Differentiation

Number of liver metastases

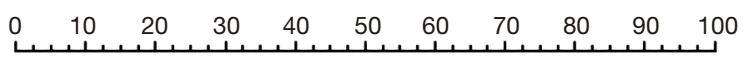

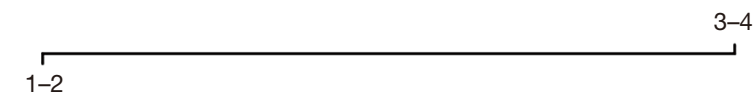

Total Points
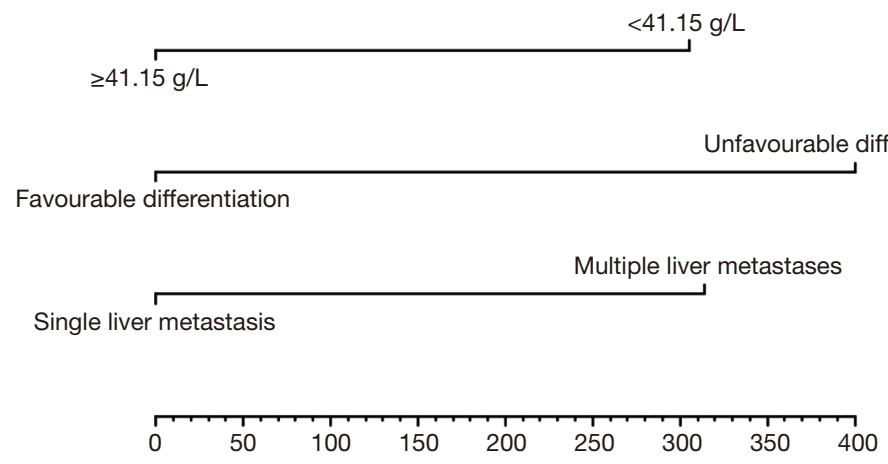

Possibility of lymph node metastasis

\begin{tabular}{llllllll}
\hline 0.2 & 0.3 & 0.4 & 0.5 & 0.6 & 0.7 & 0.8 & 0.9
\end{tabular}

Figure 1 Nomogram predicting the probability of the presence of lymph node metastasis in patients with CRLM. CRLM, colorectal cancer liver metastases; ASA, American Society of Anesthesiologists; ALB, albumin.

A

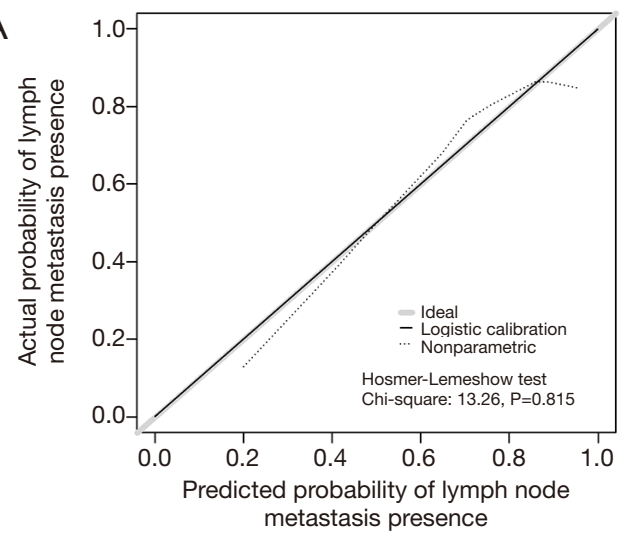

B

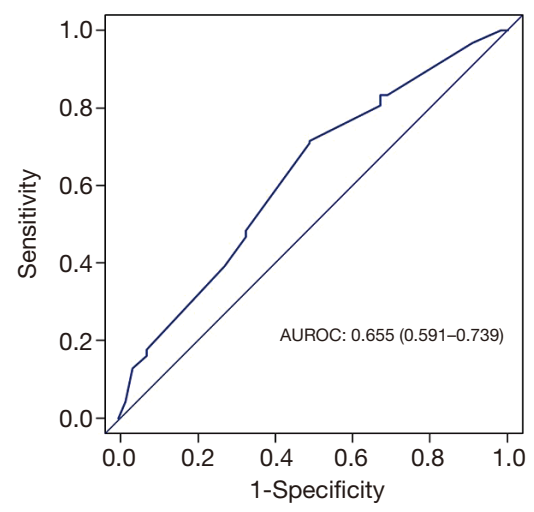

Figure 2 Assessment for nomogram in the prediction of lymph node metastasis in patients with CRLM. (A) Calibration curves. (B) ROC curves of the nomogram. CRLM, colorectal cancer liver metastases; ROC, receiver operating characteristic; AUROC, area under the receiver operating characteristic curve.

preoperative testing markers to predict primary lymph node metastasis and prognosis in patients with synchronous CRLM with simultaneous resection of CRC and liver metastases. Each independent factor has a different risky weight in the nomogram to quantify and specify when clinical strategies are determined for individual management and therapy.

Positive primary lymph node metastasis is a risky independent predictor for survival in CRLM patients. Compared with patients with CRLM without primary lymph node metastasis, patients with CRLM with lymph node metastasis have more unfavourable survival $(10,11,20)$. Nanji et al. (20) demonstrated that OS at 5 years for positive $v s$. negative nodes was $21 \%$ vs. $42 \%(\mathrm{P}=0.003)$, and cancer-specific survival was $10 \%$ vs. $43 \%(\mathrm{P}<0.001)$. It is very important to predict primary lymph node metastasis 
Table 3 Prognostic factors for positive lymph node metastasis in CRLM patients

\begin{tabular}{|c|c|c|c|c|}
\hline Factor & \multicolumn{2}{|c|}{ Univariate analysis } & \multicolumn{2}{|c|}{ Multivariate analysis } \\
\hline Age $\geq 60$ years & 0.120 & $0.634(0.357-1.126)$ & - & - \\
\hline Male & 0.380 & $1.301(0.723-2.341)$ & - & - \\
\hline $\mathrm{BMI} \geq 24 \mathrm{~kg} / \mathrm{m}^{2}$ & 0.053 & $0.564(0.316-1.008)$ & - & - \\
\hline ASA score 3-4 & 0.083 & $2.156(0.905-5.136)$ & 0.015 & $3.214(1.251-8.257)$ \\
\hline $\mathrm{CEA} \geq 10 \mathrm{ng} / \mathrm{mL}$ & 0.817 & $0.937(0.527-1.658)$ & - & - \\
\hline Preoperative $A L T \geq 18 \mathrm{U} / \mathrm{L}$ & 0.459 & $0.805(0.453-1.430)$ & - & - \\
\hline Preoperative AST $\geq 21 \mathrm{U} / \mathrm{L}$ & 0.121 & $0.632(0.354-1.128)$ & - & - \\
\hline Preoperative LDH $\geq 202.5 \mathrm{U} / \mathrm{L}$ & 0.946 & $0.978(0.518-1.849)$ & - & - \\
\hline Preoperative urea $\geq 4.6$ & 0.459 & $0.805(0.453-1.430)$ & - & - \\
\hline Preoperative creatinine $\geq 67 \mu \mathrm{mol} / \mathrm{L}$ & 0.710 & $0.897(0.505-1.592)$ & - & - \\
\hline NAC & 0.965 & $0.987(0.555-1.756)$ & - & - \\
\hline Primary site in colon & 0.994 & $0.998(0.558-1.786)$ & - & - \\
\hline Right hemicolon & 0.577 & $0.818(0.405-1.653)$ & - & - \\
\hline Poor differentiation & 0.027 & $2.406(1.106-5.233)$ & 0.005 & $3.252(1.432-7.382)$ \\
\hline T3-T4 stage & 0.115 & $2.292(0.816-6.437)$ & - & - \\
\hline
\end{tabular}

CRLM, colorectal cancer liver metastases; ASA, American Society of Anesthesiologists; CEA, carcinoembryonic antigen; ALT, alanine aminotransferase; AST, aspartate aminotransferase; ALB, albumin; FIB, fibrinogen; LDH, lactate dehydrogenase; NAC, neoadjuvant chemotherapy.

status and to schedule the appropriate individual treatment strategies before resection. This study first investigated the independent predictors of primary lymph node metastasis and then constructed the predictive nomogram. The predictors, ASA score 3-4, preoperative ALB $<41.15 \mathrm{~g} / \mathrm{L}$, poor differentiation and multiple liver metastases were independently associated with positive primary lymph node metastasis. Poor differentiation and multiple liver metastases represented aggressive biological behaviour and high tumour load, respectively (21). Thus, the relationship between two factors and primary lymph node metastasis can be predicted. It is very interesting that this study revealed that preoperative decreased ALB level was an independently risky factor for primary lymph node metastasis. Wang et al. (22) revealed that Early decrease in postoperative serum ALB predicts severe complications in patients with CRC after curative laparoscopic surgery. The mechanisms of the association between ALB level and primary lymph node metastasis may be as follows: inflammatory cytokines, such as interleukin (IL)-1b, multifunctional cytokine IL-6 and tumour necrosis factor $\alpha$, are released and induce a significant decrease in the level of ALB. These inflammatory cytokines contribute to the growth of cancer cells, micrometastases, promotion of angiogenesis and damage 
Table 4 Prognostic factors for PFS in CRLM patients after liver resection

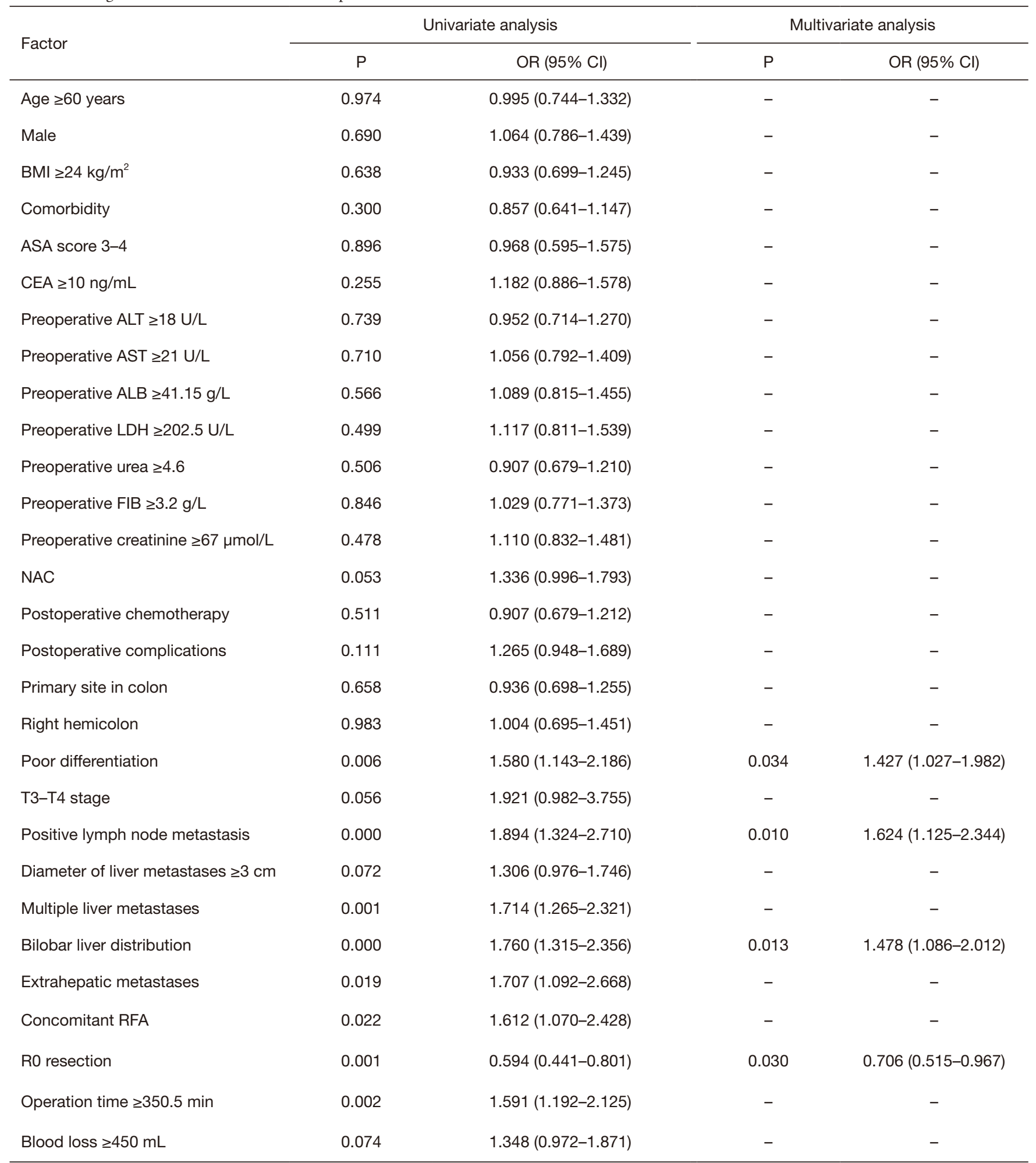

PFS, progression-free survival; CRLM, colorectal cancer liver metastases; ASA, American Society of Anesthesiologists; CEA, carcinoembryonic antigen; ALT, alanine aminotransferase; AST, aspartate aminotransferase; ALB, albumin; LDH, lactate dehydrogenase; FIB, fibrinogen; NAC, neoadjuvant chemotherapy; RFA, radiofrequency ablation. 


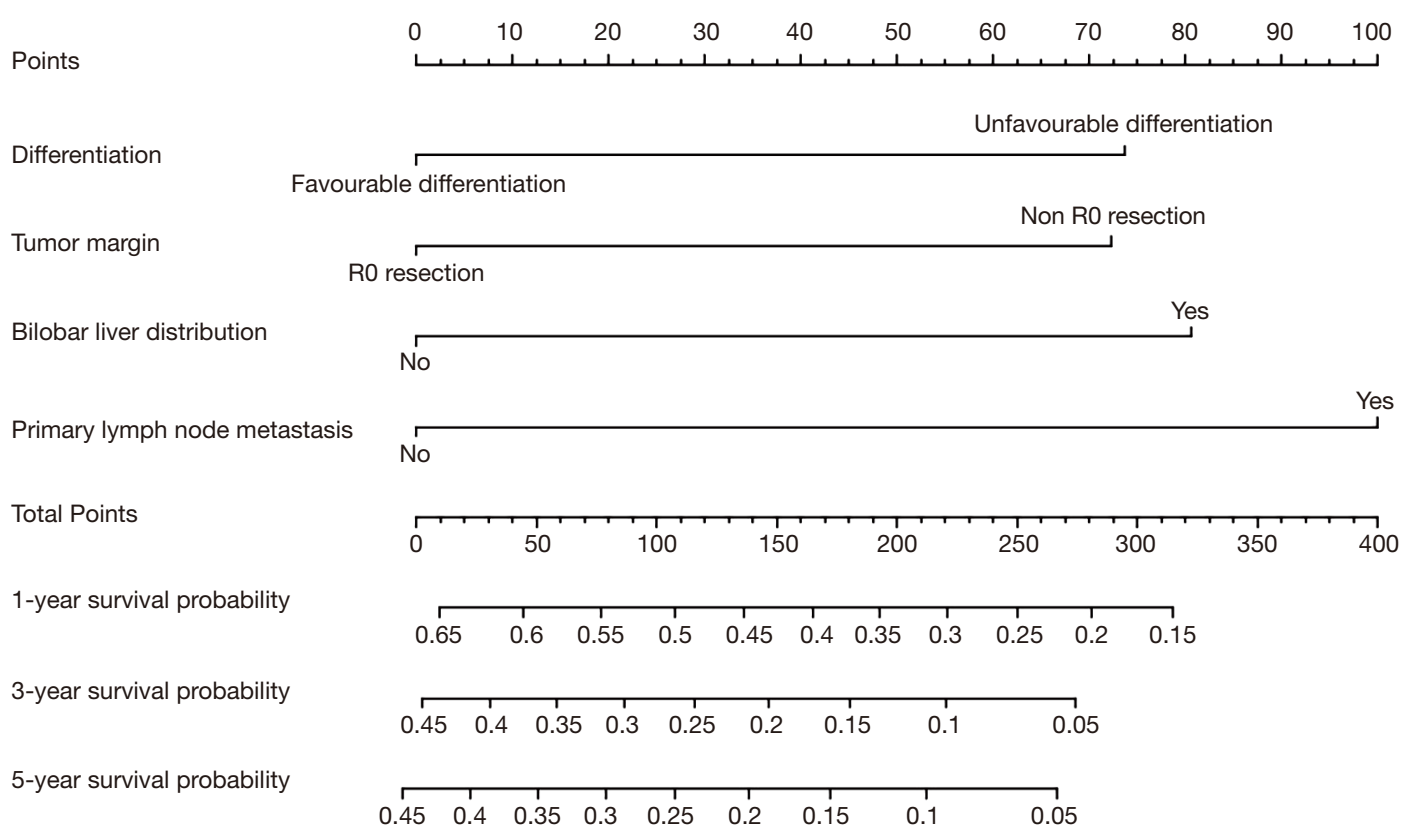

Figure 3 Nomogram for PFS. The sum of the scores for each variable was plotted on the total points axis. PFS, progression-free survival.
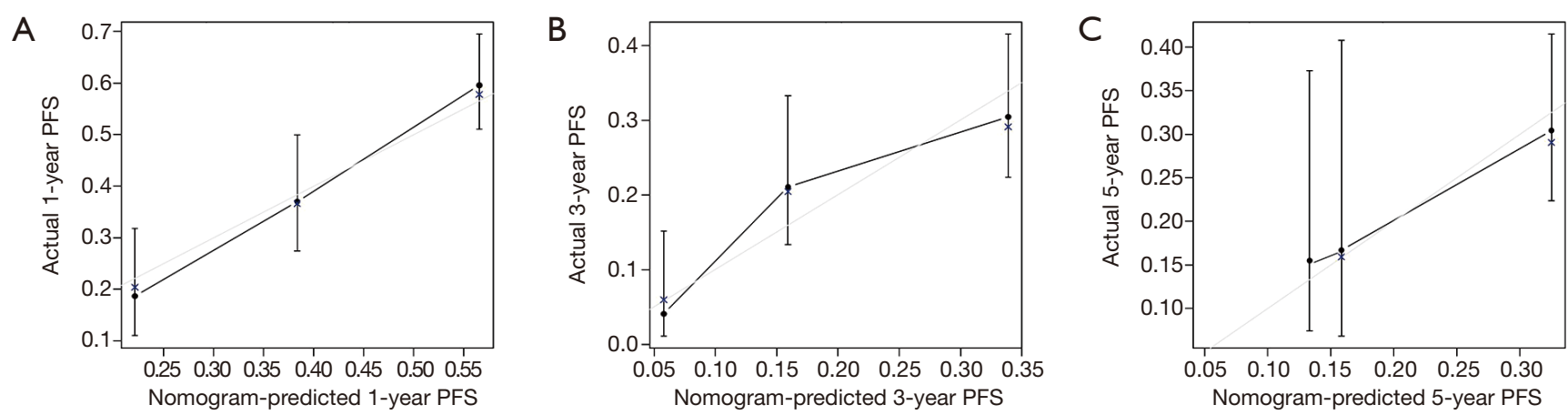

Figure 4 Calibration curves for nomogram in the prediction of PFS. Predicted survival produced by the nomogram is plotted on the $\mathrm{x}$-axis, and actual survival is plotted on the y-axis. (A) Calibration curves for 1-year PFS. (B) Calibration curves for 3-year PFS. (C) Calibration curves for 5-year PFS. PFS, progression-free survival.

to DNA (23-25). In addition, inflammatory cytokines have been reported to be associated with decreased cell-mediated immunity (26). The decreased ALB level was a symbol of the release of a large number of inflammatory factors. For patients with malignancy, the development of inflammatory response is closely associated with decreased ALB and total lymphocyte count $(27,28)$. Serum ALB is reported to act various roles, including cell growth stabilization, DNA replication, sex hormone homeostasis maintaining, and systemic inflammation modulation (22). A decreased level of ALB may reduce anticancer tolerance and cause tumour progression (29). The correlation between primary lymph node metastasis and decreased ALB level is reasonable.

This is the first study to investigate the independent predictors for postoperative major complications in patients with synchronous CRLM with simultaneous resection of CRC and liver metastases. Operation time $\geq 350.5 \mathrm{~min}$ and preoperative $\mathrm{LDH} \geq 202.5 \mathrm{U} / \mathrm{L}$ were independently associated with the presence of postoperative major complications. The extended operation time was associated with more intraoperative blood loss and more difficult operational practice (30). In addition, the extended 
operation time suggested that patients would remain under anaesthesia status for a long time and that this long anaesthesia status could weaken their physical condition and immune function (31). Therefore, the relationship between the major complications and extended operation time can be foreseen. This study was the first to reveal that a preoperative high LDH level was an independently risky factor for major complications. LDH is a cytoplasmic enzyme that catalyses the final step of anaerobic glycolysis and is released in the bloodstream when cell damage and cell death occur $(32,33)$. LDH serum levels are increased in many diseases, such as haemolytic anaemia, myocardial infarction, pulmonary embolism and rhabdomyolysis (34). In addition, it has been revealed that $\mathrm{LDH}$ promotes tumour growth, proliferation and the metastatic potential of neoplastic cells (32). Some studies have demonstrated that for CRC patients, serum levels of LDH were increased in cases of T4, N2 and M1 tumours and were related to poorer survival $(35,36)$. Therefore, the association between preoperative increased $\mathrm{LDH}$ level and postoperative major complications was reasonable. Because there were only two predictive factors of major complications, a nomogram based on these two factors has not been established.

This study also established nomograms for the prediction of PFS in patients with synchronous CRLM with simultaneous resection of CRC and liver metastases. Regarding the independent prognostic factors in the nomograms, poor differentiation, positive lymph node metastasis, bilobar liver distribution and R0 resection were consistent with the findings of previous studies. Many studies pointed to a crucial correlation between clinical prognosis and the factor of poor differentiation of CRLM (37), and analogously from our study, we showed that poor differentiation was associated with an unfavourable PFS. The reason for the PFS reduction may be because poorly differentiated or de-differentiated tumours are more likely to invade neural and vascular structures and, hence, cross histological boundaries. In addition, Hodgson et al. (10) and Nakaseko et al. (11) similarly reported that patients with lymph node metastasis were independently associated with worse survival, which corresponded to our research results. Lymph node metastasis is related to tumour heterogeneity: the higher the level of lymph node metastasis, the higher the tumour heterogeneity. Consequently, the outcome of patients with CRLM can be determined by assessing the optimal cut-off value for the number of positive lymph nodes. Additionally, a study of CRLM following hepatic resection suggested that bilobar liver distribution was a common independent and significant predictor of PFS and OS (38). The bilobar liver distribution tends to have a heavy tumour load and a wider range of surgical resection and, therefore, can cause more serious damage to liver function. Generally, the relationship between the above independent prognostic factors and PFS was reasonable.

The present study has several limitations. First, this was a retrospective study in a single centre; thus, multicentre studies should be performed to provide stronger evidence. Second, further studies should be conducted to explore the underlying mechanisms between LDH, ALB and cancer biology. Third, the examination of KRAS status was expensive and is not currently covered by medical insurance in China. Therefore, the KRAS status was only available in $51.04 \%$ patients and was not incorporated in the present analysis.

In conclusion, this study investigated the independent factors for lymph node metastasis, postoperative major complications and PFS, specifically considering preoperative testing markers in patients with synchronous CRLM with simultaneous resection of CRC and liver metastases. Based on these factors, nomograms were constructed and proved to have good calibration and discrimination, which would be helpful for clinicians to make individualized predictions and to provide specific treatment recommendations.

\section{Acknowledgments}

Funding: This study was supported by the State Key Project on Infection Diseases of China (grant No. 2017ZX10201021-007-003), The Capital Health Research and Development of Special (2018-1-4021), the National Natural Science Foundation of China (81672461, 81972311), the Non-profit Central Research Institution Fund of Chinese Academy of Medical Sciences (2019PT310026), Sanming Project of Medicine in Shenzhen (No. SZSM202011010).

\section{Footnote}

Reporting Checklist: The authors have completed the TRIPOD reporting checklist. Available at http://dx.doi. org/10.21037/apm-20-2303

Data Sharing Statement: Available at http://dx.doi. org/10.21037/apm-20-2303

Conflicts of Interest: All authors have completed the ICMJE 
uniform disclosure form (available at http://dx.doi. org/10.21037/apm-20-2303). The authors have no conflicts of interest to declare.

Ethical Statement: The authors are accountable for all aspects of the work in ensuring that questions related to the accuracy or integrity of any part of the work are appropriately investigated and resolved. The study was conducted in accordance with the Declaration of Helsinki (as revised in 2013). Ethical approval was obtained from the Institutional Review Board of the Cancer Hospital, Chinese Academy of Medical Sciences (ID:NCC2019C-016). Informed consents have been obtained.

Open Access Statement: This is an Open Access article distributed in accordance with the Creative Commons Attribution-NonCommercial-NoDerivs 4.0 International License (CC BY-NC-ND 4.0), which permits the noncommercial replication and distribution of the article with the strict proviso that no changes or edits are made and the original work is properly cited (including links to both the formal publication through the relevant DOI and the license). See: https://creativecommons.org/licenses/by-nc-nd/4.0/.

\section{References}

1. Bray F, Ferlay J, Soerjomataram I, et al. Global cancer statistics 2018: GLOBOCAN estimates of incidence and mortality worldwide for 36 cancers in 185 countries. CA Cancer J Clin 2018;68:394-424.

2. Van Cutsem E, Nordlinger B, Adam R, et al. Towards a pan-European consensus on the treatment of patients with colorectal liver metastases. Eur J Cancer 2006;42:2212-21.

3. Manfredi S, Lepage C, Hatem C, et al. Epidemiology and management of liver metastases from colorectal cancer. Ann Surg 2006;244:254-9.

4. Engstrand J, Nilsson H, Strömberg C, et al. Colorectal cancer liver metastases - a population-based study on incidence, management and survival. BMC Cancer 2018; $18: 78$.

5. Boudjema K, Locher C, Sabbagh C, et al. Simultaneous Versus Delayed Resection for Initially Resectable Synchronous Colorectal Cancer Liver Metastases: A Prospective, Open-label, Randomized, Controlled Trial. Ann Surg 2021;273:49-56.

6. Wang J, Griffiths C, Simunovic M, et al. Simultaneous versus staged resection for synchronous colorectal liver metastases: A population-based cost analysis in Ontario,
Canada - Health economic evaluation. Int J Surg 2020;78:75-82.

7. Osada S, Imai H, Sasaki Y, et al. Strategy for synchronous and multiple liver metastasis. Hepatogastroenterology 2012;59:198-203.

8. Zhang YF, Mao R, Chen X, et al. Prognostic Analysis of 102 Patients with Synchronous Colorectal Cancer and Liver Metastases Treated with Simultaneous Resection. Chin Med J (Engl) 2017;130:1283-9.

9. Bizzoca C, Delvecchio A, Fedele S, et al. Simultaneous Colon and Liver Laparoscopic Resection for Colorectal Cancer with Synchronous Liver Metastases: A Single Center Experience. J Laparoendosc Adv Surg Tech A 2019;29:934-42.

10. Hodgson R, Sethi H, Ling AH, et al. Combined hepatectomy and hepatic pedicle lymphadenectomy in colorectal liver metastases is justified. HPB (Oxford) 2017;19:525-9.

11. Nakaseko Y, Haruki K, Shiba H, et al. Impact of fresh frozen plasma transfusion on postoperative inflammation and prognosis of colorectal liver metastases. J Surg Res 2018;226:157-65.

12. Tang M, Wang $\mathrm{H}$, Cao $\mathrm{Y}$, et al. Nomogram for predicting occurrence and prognosis of liver metastasis in colorectal cancer: a population-based study. Int J Colorectal Dis 2021;36:271-82.

13. Yan Y, Liu H, Mao K, et al. Novel nomograms to predict lymph node metastasis and liver metastasis in patients with early colon carcinoma. J Transl Med 2019;17:193.

14. Jansen MC, van Wanrooy S, van Hillegersberg R, et al. Assessment of systemic inflammatory response (SIR) in patients undergoing radiofrequency ablation or partial liver resection for liver tumors. Eur J Surg Oncol 2008;34:662-7.

15. Liu Y, Chen S, Zheng C, et al. The prognostic value of the preoperative c-reactive protein/albumin ratio in ovarian cancer. BMC Cancer 2017;17:285.

16. Liu Z, Jin K, Guo M et al. Prognostic Value of the CRP/Alb Ratio, a Novel Inflammation-Based Score in Pancreatic Cancer. Ann Surg Oncol 2017;24:561-8.

17. Huang H, Zhang L, Chen DB, et al. Validation of Prognosis Value of Cumulative Prognostic Scores Based on Serum High-Density Lipoprotein Cholesterol and Albumin Levels in Patients with Colorectal Cancer. J Cancer 2019;10:35-42.

18. Diagnosis And Treatment Guidelines For Colorectal Cancer Working Group CSOCOC. Chinese Society of Clinical Oncology (CSCO) diagnosis and treatment 
guidelines for colorectal cancer 2018 (English version). Chin J Cancer Res 2019;31:117-34.

19. Li C, Chen J, Qin G. Partial Youden index and its inferences. J Biopharm Stat 2019;29:385-99.

20. Nanji S, Tsang ME, Wei X, et al. Regional lymph node involvement in patients undergoing liver resection for colorectal cancer metastases. Eur J Surg Oncol 2017;43:322-9.

21. Ma ZH, Wang YP, Zheng WH, et al. Prognostic factors and therapeutic effects of different treatment modalities for colorectal cancer liver metastases. World J Gastrointest Oncol 2020;12:1177-94.

22. Wang $Y$, Wang $H$, Jiang J, et al. Early decrease in postoperative serum albumin predicts severe complications in patients with colorectal cancer after curative laparoscopic surgery. World J Surg Oncol 2018;16:192.

23. Yang J, Wezeman M, Zhang X, et al. Human C-reactive protein binds activating Fcgamma receptors and protects myeloma tumor cells from apoptosis. Cancer Cell 2007;12:252-65.

24. Nazha B, Moussaly E, Zaarour M, et al. Hypoalbuminemia in colorectal cancer prognosis: Nutritional marker or inflammatory surrogate? World J Gastrointest Surg 2015;7:370-7.

25. Haruki K, Shiba H, Horiuchi T, et al. Impact of the C-reactive protein to albumin ratio on long-term outcomes after hepatic resection for colorectal liver metastases. Am J Surg 2017;214:752-6.

26. Shalapour S, Karin M. Immunity, inflammation, and cancer: an eternal fight between good and evil. J Clin Invest 2015;125:3347-55.

27. Erdman SE, Poutahidis T. Roles for inflammation and regulatory $T$ cells in colon cancer. Toxicol Pathol 2010;38:76-87.

28. Crumley AB, Stuart RC, McKernan M, et al. Is hypoalbuminemia an independent prognostic factor in patients with gastric cancer? World J Surg

Cite this article as: Yao J, Chen Q, Deng Y, Zhao J, Bi X, Li Z, Huang Z, Zhang Y, Zhou J, Zhao H, Cai J. Nomograms predicting primary lymph node metastases and prognosis for synchronous colorectal liver metastasis with simultaneous resection of colorectal cancer and liver metastases. Ann Palliat Med 2021;10(4):4220-4231. doi: 10.21037/apm-20-2303
2010;34:2393-8.

29. Li G, Gao J, Liu ZG, et al. Influence of pretreatment ideal body weight percentile and albumin on prognosis of nasopharyngeal carcinoma: Long-term outcomes of 512 patients from a single institution. Head Neck 2014;36:660-6.

30. Margonis GA, Kim Y, Samaha M, et al. Blood loss and outcomes after resection of colorectal liver metastases. J Surg Res 2016;202:473-80.

31. Serrano PE, Gafni A, Parpia S, et al. Simultaneous resection of colorectal cancer with synchronous liver metastases (RESECT), a pilot study. Int J Surg Protoc 2018;8:1-6.

32. Augoff K, Hryniewicz-Jankowska A, Tabola R. Lactate dehydrogenase 5: an old friend and a new hope in the war on cancer. Cancer Lett 2015;358:1-7.

33. Storey KB. Comparative enzymology-new insights from studies of an "old" enzyme, lactate dehydrogenase. Comp Biochem Physiol B Biochem Mol Biol 2016;199:13-20.

34. Kostakis ID, Vaiopoulos AG, Garoufalia Z, et al. What can preoperative blood tests tell us about colorectal cancer? J buon 2018;23:84-95.

35. Kostakis ID, Vaiopoulos AG, Philippou A, et al. Preoperative serum lactate dehydrogenase levels in colorectal and gastric cancer: a hospital-based case-control study. Biomark Med 2013;7:131-7.

36. Li G, Wang Z, Xu J, et al. The prognostic value of lactate dehydrogenase levels in colorectal cancer: a meta-analysis. BMC Cancer 2016;16:249.

37. Jo HS, Kim DS, Jung SW, et al. Clinical significance of post-hepatectomy hepatic failure in patients with liver metastases from colorectal cancer. Ann Hepatobiliary Pancreat Surg 2018;22:93-100.

38. Haruki K, Shiba H, Fujiwara Y, et al. Preoperative peripheral blood neutrophil count predicts long-term outcomes following hepatic resection for colorectal liver metastases. Oncol Lett 2017;13:3688-94. 